\title{
A deep learning algorithm to detect coronavirus (COVID-19) disease using CT images
}

\author{
Mojtaba Mohammadpoor ${ }^{\text {Corresp., }}{ }^{1}$, Mehran Sheikhi karizaki $^{2}$, Mina Sheikhi karizaki ${ }^{3}$ \\ 1 Electrical and Computer Department, University of Gonabad, Gonabad, Iran \\ 2 Electrical engineering Dept., University of Birjand, Birjand, Iran \\ 3 Nursing Department, Islamic Azad University of Sabzevar, Sabzevar, Iran \\ Corresponding Author: Mojtaba Mohammadpoor \\ Email address: mohammadpur@gonabad.ac.ir
}

Background. COVID-19 pandemic imposed a lockdown situation to the world these months. It faced the around globe researchers and scientists serious efforts from its detection to its treatment.

Methods. Pathogenic laboratory testing is the gold standard but it is time-consuming. Lung CT-scans and X-rays are of the other common methods applied by researchers to detect COVID-19 positive cases. In this paper, we propose a deep learning neural network-based model as an alternative fast screening method that can be used for detecting the COVID-19 cases by analyzing CT-scans.

Results. Applying the proposed method on a publicly available dataset collected of positive and negative cases shows its ability on distinguishing them by analyzing each individual CT image. Effect of different parameters on the performance of the proposed model is studied and tabulated. By selecting random train an test images, the overall accuracy and ROC-AUC of the proposed model can easily exceed $95 \%$ and $90 \%$, respectively without any image pre-selecting or preprocessing. 


\section{A Deep Learning Algorithm To Detect Corona Virus} (COVID-19) Disease Using CT Images

4

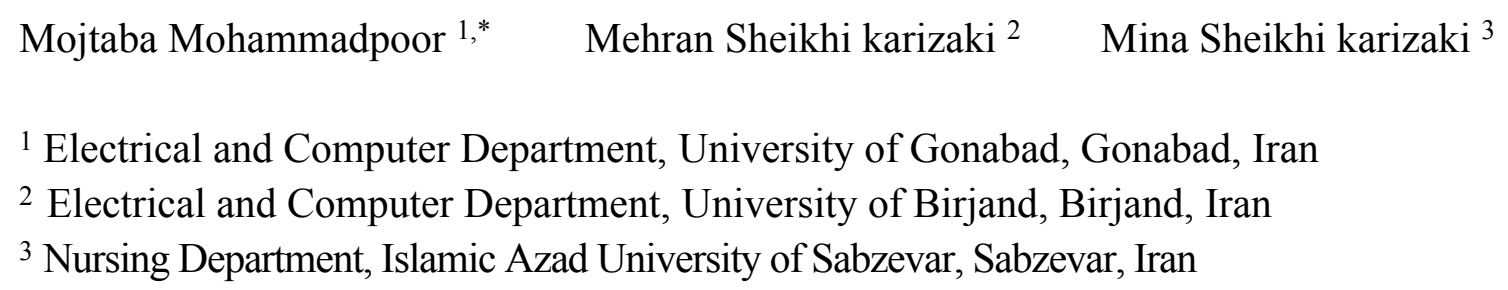

\section{Abstract}

Background. COVID-19 pandemic imposed a lockdown situation to the world these months. It faced the around globe researchers and scientists serious efforts from its detection to its treatment.

Methods. Pathogenic laboratory testing is the gold standard but it is time-consuming. Lung CTscans and X-rays are of the other common methods applied by researchers to detect COVID-19 positive cases. In this paper, we propose a deep learning neural network-based model as an alternative fast screening method that can be used for detecting the COVID-19 cases by analyzing CT-scans.

Results. Applying the proposed method on a publicly available dataset collected of positive and negative cases shows its ability on distinguishing them by analyzing each individual CT image. Effect of different parameters on the performance of the proposed model is studied and tabulated. By selecting random train an test images, the overall accuracy and ROC-AUC of the proposed model can easily exceed $95 \%$ and $90 \%$, respectively without any image pre-selecting or preprocessing.

\section{Introduction}

Severe acute respiratory syndrome coronavirus 2 (SARS-CoV-2) simply called as corona virus or COVID-19 is currently one the most life-threatening problems around the world. Coronavirus disease 2019 (COVID-19) is a highly infectious disease caused by severe acute respiratory syndrome coronavirus 2 (Wang, Chen et al., 2020). The disease first originated in 31 December 2019 from Wuhan, Hubei Province, China and since then it has spread globally across the world. The cumulative incidence of the causative virus (SARS-CoV-2) is rapidly increasing and has affected 196 countries and territories and on 4 May 2020, a total of 3,581,884 confirmed positive cases have been reported leading to 248,558 deaths (Coronavirus - worldometer). The impact is 
40 such that the World Health Organization (WHO) has declared the ongoing pandemic of COVID4119 a public health emergency of international concern (Daksh Trehan, 2020).

42 Pandemic caused by COVID-19 has major difference by other related viruses, such as Middle 43 East Respiratory Syndrome (MERS) and Severe Acute Respiratory Syndrome (SARS), which is 44 its ability to spread rapidly through human contact and leave nearly $20 \%$ infected subjects as 45 symptom-less carriers (Mallapaty, Smriti,2020).

46 Pathogenic laboratory testing is the gold standard but it is time-consuming, therefore, other 47 diagnostic methods are needed to detect the disease in a timely manner. COVID-19 makes some 48 changes in CT images. Mahmoud, H., Taha, M.S., Askoura, A. et al. (Mahmoud, H. et al.,2020) have analyzed recent reports and stated that the sensitivity of RT-PCR in diagnosing COVID-19 is $71 \%$ while sensitivity of CT is $98 \%$. It is possible that small changes in CT images may be neglected during visual inspection and we hypothesized that an Artificial Intelligence's method might be able to detect COVID-19's positive cases and provide a clinical diagnosis ahead of the pathogenic test, thus saving critical time for disease control. The main contribution of this paper is to propose a prediction mode based on convolutional neural network (CNN) deep learning method, which is able to be trained by some CT images of corona virus infected lungs and CT images of healthy lungs. The trained model is then able to classify any new CT image as positive and negative COVID-19 at a faster speed.

\section{Related works}

Several efforts have performed by researchers in detecting coronavirus affected cases using radio graphical images. Alibaba has developed AI solutions to predict the duration, size and peak of the outbreak, which is tested in real world in various regions of China and claimed to have $98 \%$ accuracy (C. Huang et al.,2020). As the COVID-19 virus affects the lungs of peoples, some deep learning studies have proposed to detected the disease by processing chest X-ray and CT images of lung (Toğaçar et al.,2019). A deep learning model for detecting pneumonia is proposed in (Stephen, Okeke et al.,2019). Their suggested model is consisted of convolution layers, dense blocks, and flatten layers. Their input image size is $200 * 200$ pixels. Their final success rate is $93.73 \%$.

Chouhan et al. in (Chouhan, Vikash et al.,2020) have proposed a deep learning model for classifying the pneumonia images into three classes, namely: bacterial pneumonia, virus pneumonia, and normal images. In the first step, they proposed some preprocessing methods to remove noise from the images. Then, they applied an augmentation technique on the images before using them for training their model. Their overall classification accuracy is $96.39 \%$. Authors in (www.medrxiv.org, 2020) used pathogen-confirmed COVID-19 cases (325 images) and 740 images diagnosed with typical viral pneumonia. Their internal validation reached to an overall classification accuracy of $89.5 \%$. Their external testing dataset reached to an overall 77 accuracy of $79.3 \%$. 
78 Toğaçar et al. in (Toğaçar et al., 2020) have proposed a deep learning method to classify chest 79 X-ray images to detect corona virus infected patients. Their dataset consists of three classes, 80 namely: normal, pneumonia and coronavirus images. They achieved to $99.27 \%$ classification 81 rate.

82 Zahangir et al. in (Zahangir Alom, Md et al., 2020) have proposed a multi task deep learning 83 algorithm for this purpose. They have used and compared CT scan and X-ray images in their 84 model. They achieved around $84.67 \%$ testing accuracy from X-ray images and $98.78 \%$ accuracy 85 in CT-images, meaning that $\mathrm{CT}$ scan images are more accurate. They have also tried to 86 determine the percentage of infected regions in $\mathrm{CT}$ and X-ray images.

87 Zheng et al. in (Zheng $C$ et al., 2020) have proposed a 3D deep neural network to predict the 88 probability of COVID-19 infectious. They have used 499 CT volumes for training and $131 \mathrm{CT}$ 89

\section{Materials \& Methods}

Artificial intelligence improves the representations needed for pattern recognition using a machine composed of multiple layers, uses raw data as input (Goodfellow I et al.,2016). Deep learning is a semi-supervised technique for labeling datasets. For instance, if a deep network is fed with several tumor cells, it can interpret an image to detect insignificant aspects ( $L i$ Y., 2017). Since the last few years, deep learning techniques completely changed the scenario of many research fields by promising results with highest accuracy, especially, in medical image processing fields, such as retina image, chest X-ray, and brain MRI images(M. Mahmud et al. ,2018; I. W. Harsono, S. Liawatimena, T. W. Cenggoro, 2020).

\section{Convolutional Neural Networks (CNN)}

Among deep learning classifiers convolutional neural networks (CNN) have more usage in computer vision and medical image analysis tasks compare to others, and it is proved that it has better results. (Panwar, Harsh, et al.,2020). CNN, as other types of artificial neural network models, has multiple layers and it can process data effectively and achieve high accurate results. Convolution, pooling, flattening, and fully connected layers are consisting CNN structure 
118 (Goodfellow I et al.,2016). CNN can extract the features from the images individually, and then 119 classify them. This unique characteristic can applied on medical images and provides a great 120 support in the advancement of health community research (Choe, Jooae, et al.,2020).

$121 \mathrm{CNN}$ models have self-learning abilities helps them to achieve superior and human-like 122 classification results on multi-class problems (Ucar, Ferhat, and Deniz Korkmaz, 2020).

123

124

125

126

127

128

129

130

131

132

133

134

135

136

137

138

139

140

141

142

143

144

145

146

147

148

149

150

151

152

153

154

155

156

157

CNN models had been used in different applications and achieve amazing results (Le NQK, Nguyen, 2019). In general, they compromised of input, feature extraction and output layers. Feature extraction stage can have several repeated convolution layers, rectified linear units and pooling layers. Convolution layers could detect different patterns, such as textures, edges, shapes etc. in images (Jang Y et al., 2018, Raghu et al., 2020). They also have multilayer perceptrons (fully connected) which all neurons in each layer are connected to all neurons in the next layer. This hierarchical structure provides high-level feature maps and improved overall accuracy (Ucar, Ferhat, and Deniz Korkmaz, 2020).

\section{Data Collection}

The data used in this paper is downloaded from publicly available dataset (Rahimzadeh, Mohammad, Abolfazl Attar, and Seyed Mohammad Sakhaei, 2020). They have collected 15589 CT images of 95 positive patients and 48260 images of 282 negative persons. The pictures are 16bit tiff format and $512 * 512$ size. Each person has three folder, each folder includes some images representing a breath sequence. Fig. 1 is showing some image samples.

\section{Fig. 1: Samples of COVID-CTset images (Rahimzadeh, Mohammad, Abolfazl Attar, and Seyed Mohammad Sakhaei, 2020)}

In some images of a breath sequence, the inside of the lung is visible. In some of them (e.g. first and lost images of a sequence), inside of the lung is not clear. Fig. 2 shows some sequential images.

Fig. 2: Samples of sequential COVID-CTset images ( Rahimzadeh, Mohammad, Abolfazl Attar, and Seyed Mohammad Sakhaei, 2020)

\section{Proposed Method}

A deep learning model based on convolutional neural network (CNN) is proposed in this paper to distinguish positive and negative COVID-19 cases. In some researches some preprocessing stages are applied on images to select special images of a breath sequence or highlight lung infected area, before entering them to the classification algorithm ( Rahimzadeh, Mohammad, 
158

159

160

161

162

163

164

165

166

167

168

169

170

171

172

173

174

175

176

177

178

179

180

181

182

183

184

185

186

187

188

189

190

191

192

193

194

195

196

197

Abolfazl Attar, and Seyed Mohammad Sakhaei., 2020). In order to have a fully automated algorithm, in this paper no preprocessing, preselecting or ROI selecting is performed on the images. Fig. 3 is showing the proposed model. As it is shown, it is consisted of three steps. In each step a convolution layer (Conv) is used. It is a 2-D convolutional layer which applies sliding convolutional filters to the input image. The layer moves the filters along the input and convolves the input by them vertically and horizontally, and computes the dot product of the input and the weights, and then adds a bias term. In our proposed model, the size of used filter is selected as $3 \times 3$. The number of filters are selected as $8,16,32,64 \ldots$ for other steps.

To reduce sensitivity of CNN to network initialization and speed up its training, a batch normalization layer is used between convolutional layer and nonlinearities. It normalizes each input channel across a mini-batch.

A rectified Linear Unit (ReLU) layer is used in each step to perform a threshold operation to each element of the input, meaning that each value less than zero is set to zero.

A max pooling layer is used in each step to run down-sampling by dividing the input into rectangular pooling regions, and computing the maximum of each region.

(1)

Fig. 3: The proposed model

In order to evaluate the proposed method, cross-validation technique is performed. For this purpose, the images of each category (i.e. positive or negative) are divided into two groups, namely train, and test. Number of images in each group depends on application. In this paper, the algorithm is performed several times using different percentages. More training images imposed more processing time and leads higher accuracy. A trained network could process any individual image immediately.

\section{Results}

The original images have a size of $512 * 512$. In order to reduce the processing border, images with reduced dimensions can be used. Some training options should be defined for training the model. In this paper stochastic gradient descent with momentum (SGDM) optimizer is used. Initial learn rate is selected as 0.001 . Maximum number of epochs can affect the training time, as well as accuracy.

Because of randomly selection of train and test images, the model is launched several times. Figs. 4,5,6 are showing results of one running the algorithm. In this sample run, 50\% of images in each category are selected randomly for training the model. Others are used for evaluating it. For this purpose, a total number of 2297 and 8961 images are selected in positive and negative categories, respectively. The images are resized into $200 * 200$ and used for train the deep learning model. Seven convolution layers are used in this case, and maximum epoch is selected as 80. Confusion matrices and Receiver Operating Characteristics (ROC) of the model are shown 
198

199

200

201

202

203

204

205

206

207

208

209

210

211

212

213

214

215

216

217

218

219

220

221

222

223

224

225

226

227

228

229

230

231

232

233

234

235

236

237

in Figs. 5 and 6, for evaluating training, test and all data portions, respectively. Total accuracy and Area Under the Curve (AUC) of the ROC curves are shown in the first row of Table 1. Results of running the algorithm by other different parameters are also summarized in Table1.

Fig. 4: Training progress of running the model

Fig. 5: A) Confusion matrix definitions (Wiki. 2020), B) Confusion matrix and, C) ROC curve of evaluating training data

Fig. 6: A) Confusion matrix and, B) ROC curve of evaluating test data

Table 1: Summarized results of some runs of the proposed algorithm

\section{Discussion}

Some different parameters can affect the model performance. The first parameter is the image sizes. Large images have more details, hence it is expected to have better results, as shown upper rows in Table 1 have better results compared to lower rows. Second parameter is percentage and method in dividing dataset into train and test category. More training data normally will be caused into higher accuracy. Random selection is selected in all experiments listed in Table 1. In order to show the robustness of the model, experiment \#6 is performed over the opposite data portions of experiment $\# 5$, meaning that the model is trained by testing data of experiment $\# 5$ and then evaluated by train portion. As it shown the results are reasonable. Another parameter is the number of epochs that deep learning model is performed. More epochs will be caused to better training the model. The last parameter is the number of convolution layers. A deeper network certainly will be well trained. In case of small images, the number of convolution layers may be limited due to padding procedure. Hence, fewer convolution layers are used in $4^{\text {th }}, 5^{\text {th }}$, and $6^{\text {th }}$ experiments. While using bigger images, this limitation is removed. In the first, second and third experiments in Table 1, seven convolution layers are used. More layers are not test but it is expected that they will have better results. Another parameter is the bit numbers of the images. Originally, the images are $16 \mathrm{bit}$, in this study they changed to 8 bit.

Peer] Comput. Sci. reviewing PDF | (CS-2020:08:51801:2:0:CHECK 25 Nov 2020) 
238 Eventually, as it shown the overall accuracy and ROC-AUC of the proposed model can easily 239 exceed $95 \%$ and $90 \%$, respectively. It should be considered that in this research all CT images 240 during a breath cycle is used, since the inside lung and also infected area can be seen in just few 241 images, the accuracy rate is adequately high which makes it a robust model for detecting 242 CVID19 patients. It is expected that the accuracy increase to $100 \%$ by adjusting some 243 parameters, but these parameters can increase the model training time. From an applicable view 244 of point, the model can be trained separately in high performance computers, and then, the 245 trained model be used by doctors, because the trained model can process any individual image in 246 a moment and predict its label almost immediately.

247

248

249

250

251

252

253

254

255

256

257

258

259

260

261

262

263

264

265

266

267

268

269

270

271

272 273

274

275

276

\section{Conclusions}

Detecting CVID19 positive cases from CT scan images would be helpful for doctors to detect the patients without performing timely and costly molecular tests. In this paper a machine learning model based on deep learning is proposed for this purpose. The proposed model is evaluated by running it several times on a publicly available CT images dataset. Some percent of images are selected randomly and used for training the proposed model, while the model is evaluated using the remained images. Other adjustable parameters are also discussed. The results implies the ability of the proposed model in classification of images. The overall accuracy and ROC-AUC of the proposed model can easily exceed $95 \%$ and $90 \%$, respectively, which makes it a strong CAD tool for using by doctors.

\section{References}

C. Huang, Y. Wang, X. Li , L. Ren, J. Zhao , Y. Hu , L. Zhang, G. Fan, J. Xu , X. Gu, et al., Clinical features of patients infected with 2019 novel coronavirus in Wuhan, China, The Lancet 395 (10223) (2020) 497-506 .

Choe, Jooae, et al. "Deep learning-based image conversion of CT reconstruction kernels improves radiomics reproducibility for pulmonary nodules or masses." Radiology 292.2 (2019): 365-373.

Chouhan, Vikash, et al. "A novel transfer learning based approach for pneumonia detection in chest X-ray images." Applied Sciences 10.2 (2020): 559.

Coronavirus - worldometer, link: https://www.worldometers.info/coronavirus/, [online accessed]

Daksh Trehan, detecting-covid-19-using-deep-learning, (2020)

https://towardsdatascience.com/detecting-covid-19-using-deep-learning-262956b6f981

Peer] Comput. Sci. reviewing PDF | (CS-2020:08:51801:2:0:CHECK 25 Nov 2020) 
277 Gifani, P., Shalbaf, A. \& Vafaeezadeh, M. Automated detection of COVID-19 using ensemble of 278 transfer learning with deep convolutional neural network based on CT scans. Int J CARS (2020). 279 https://doi.org/10.1007/s11548-020-02286-w

280

281

282

283

284

285

286

287

288

289

290

291

292

293

294

295

296

297

298

299

300

301

302

303

304

305

306

307

308

309

310

311

312

313 Mallapaty, Smriti. "What the cruise-ship outbreaks reveal about COVID-19." Nature 580.7801 314 (2020): 18-18.

315

Goodfellow I, Bengio Y, Courville A, Bengio Y. Deep Learning. MIT press Cambridge; 2016.

https://www.medrxiv.org/content/10.1101/2020.02.14.20023028v5, preprint

I.M. Baltruschat, H. Nickisch, M. Grass, T. Knopp, A. Saalbach, Comparison of deep learning approaches for multi-label chest X-ray classification, Sci. Rep. 9 (2019) 6381, https://doi.org/10.1038/s41598-019-42294-8

I. W. Harsono, S. Liawatimena, T. W. Cenggoro, Lung nodule detection and classification from thorax ct-scan using retinanet with transfer learning, Journal of King Saud University-Computer and Information Sciences (2020).

Jaiswal, Amit Kumar, et al. "Identifying pneumonia in chest X-rays: A deep learning approach." Measurement 145 (2019): 511-518.

Jang Y, Kim S, Kim K, Lee D. 2018. Deep learning-based classification with improved time resolution for physical activities of children. PeerJ 6:e5764 https://doi.org/10.7717/peerj.5764

Le NQK, Nguyen V. 2019. SNARE-CNN: a 2D convolutional neural network architecture to identify SNARE proteins from high-throughput sequencing data. PeerJ Computer Science 5:e177 https://doi.org/10.7717/peerj-cs. 177

Li Y. Deep Reinforcement Learning: An Overview arXiv preprint arXiv:1701.07274.2017.

M. Mahmud, M. S. Kaiser, A. Hussain, S. Vassanelli, Applications of deep learning and reinforcement learning to biological data, IEEE transactions on neural networks and learning systems 29 (2018) 2063-2079.

Mahmoud, H., Taha, M.S., Askoura, A. et al. Can chest CT improve sensitivity of COVID-19 diagnosis in comparison to PCR? A meta-analysis study. Egypt J Otolaryngol 36, 49 (2020). https://doi.org/10.1186/s43163-020-00039-9 
316 Mukherjee, H., Ghosh, S., Dhar, A. et al. Deep neural network to detect COVID-19: one 317 architecture for both CT Scans and Chest X-rays. Appl Intell (2020).

318 https://doi.org/10.1007/s10489-020-01943-6

319

320

321

322

323

324

325

326

327

328

329

330

331

332

333

334

335

336

337

338

339

340

341

342

343

344

345

346

347

348

349

350

351

352

353

Panwar, Harsh, et al. "Application of Deep Learning for Fast Detection of COVID-19 in X-Rays using nCOVnet." Chaos, Solitons \& Fractals (2020): 109944.

Raghu, Shivarudhrappa, et al. "EEG based multi-class seizure type classification using convolutional neural network and transfer learning." Neural Networks 124 (2020): 202-212.

Rahimzadeh, Mohammad, Abolfazl Attar, and Seyed Mohammad Sakhaei. "A Fully Automated Deep Learning-based Network For Detecting COVID-19 from a New And Large Lung CT Scan Dataset." medRxiv (2020). Datdaset: https://github.com/mr7495/COVID-CTset

Stephen, Okeke, et al. "An efficient deep learning approach to pneumonia classification in healthcare." Journal of healthcare engineering 2019 (2019).

Toğaçar, Mesut, Burhan Ergen, and Zafer Cömert. "COVID-19 detection using deep learning models to exploit Social Mimic Optimization and structured chest X-ray images using fuzzy color and stacking approaches." Computers in Biology and Medicine (2020): 103805.

Wang, Chen, et al. "A novel coronavirus outbreak of global health concern." The Lancet 395.10223 (2020): 470-473.

Wiki, https://en.wikipedia.org/wiki/Confusion_matrix, retrieved Oct 19, 2020.

Zahangir Alom, Md, et al. "COVID_MTNet: COVID-19 Detection with Multi-Task Deep Learning Approaches." arXiv (2020): arXiv-2004.

Zheng C, Deng X, Fu Q, Zhou Q, Feng J, Ma H, Liu W, Wang X. Deep learning-based detection for COVID-19 from chest CT using weak label. medRxiv. 2020 Jan 1.

Ucar, Ferhat, and Deniz Korkmaz. "COVIDiagnosis-Net: Deep Bayes-SqueezeNet based Diagnostic of the Coronavirus Disease 2019 (COVID-19) from X-Ray Images." Medical Hypotheses (2020): 109761.

Peer] Comput. Sci. reviewing PDF | (CS-2020:08:51801:2:0:CHECK 25 Nov 2020) 
Figure 1

Samples of COVID-CTset images (Rahimzadeh, Mohammad, Abolfazl Attar, and Seyed Mohammad Sakhaei, 2020)

\section{Samples from dataset}

\section{COVID-19}
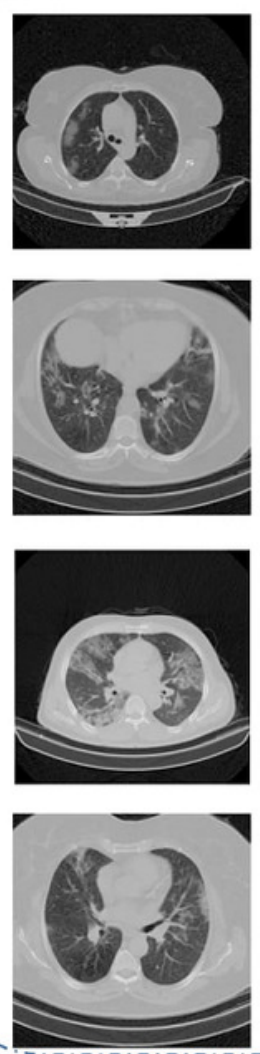
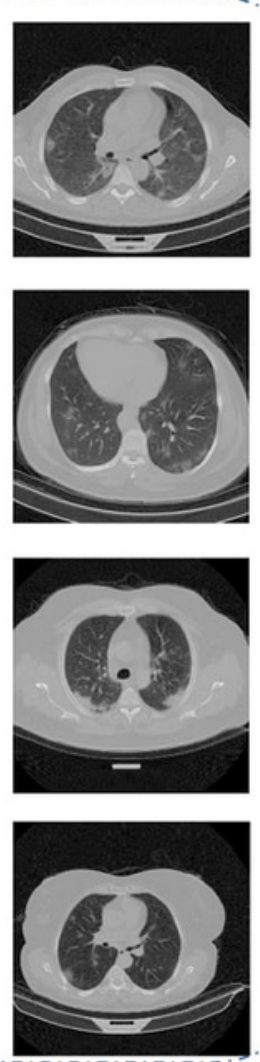

Normal
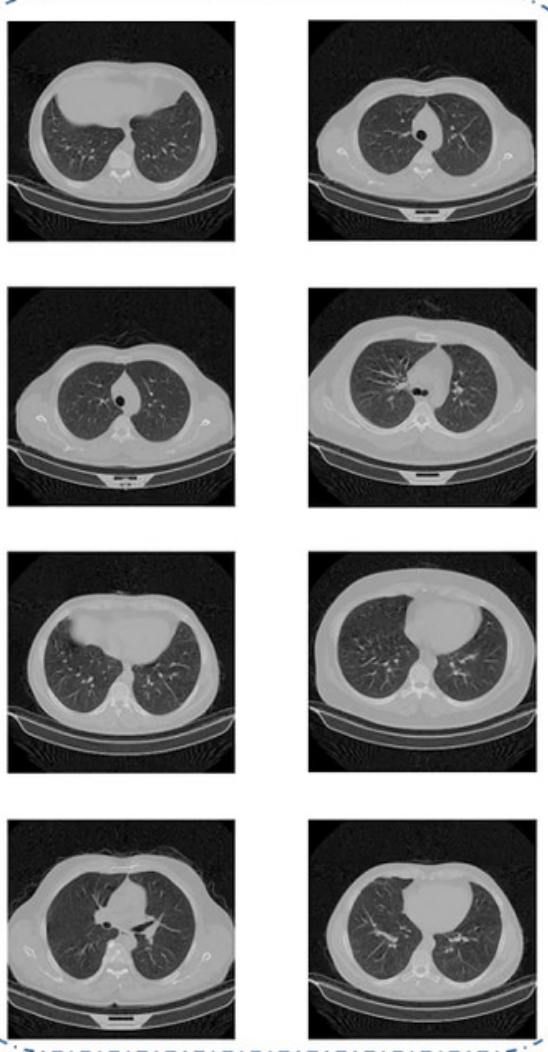


\section{Figure 2}

Samples of sequential COVID-CTset images ( Rahimzadeh, Mohammad, Abolfazl Attar, and Seyed Mohammad Sakhaei, 2020)

First images of a sequence
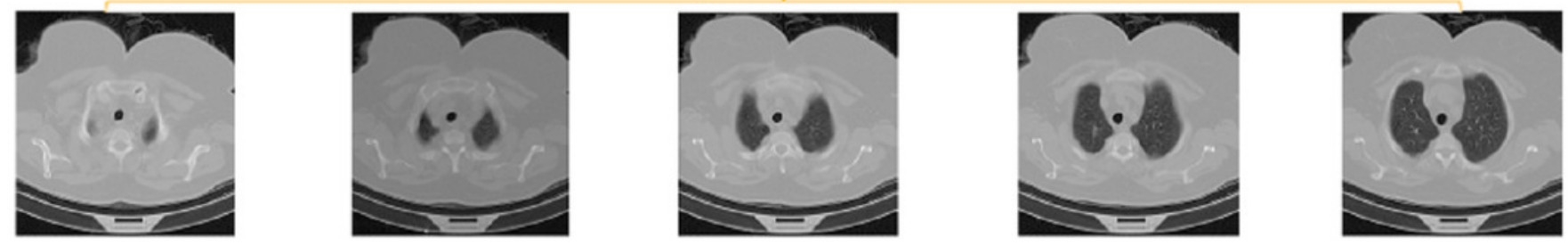

Middle images of a sequence
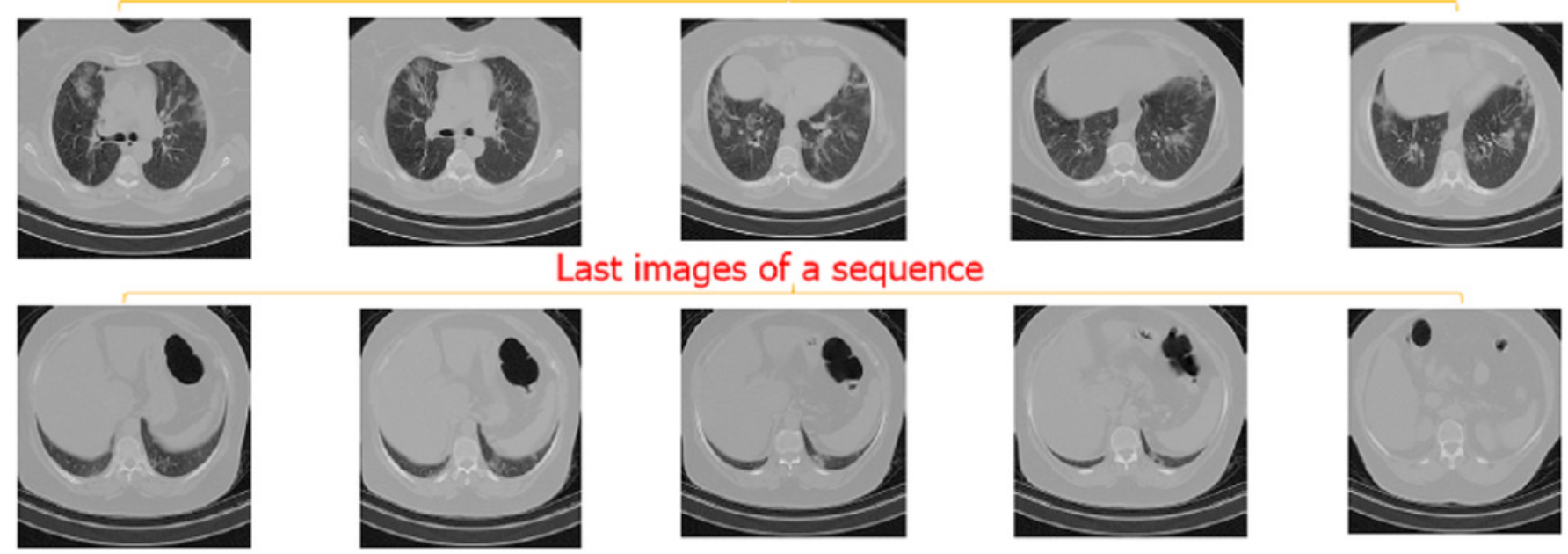
Figure 3

The proposed model

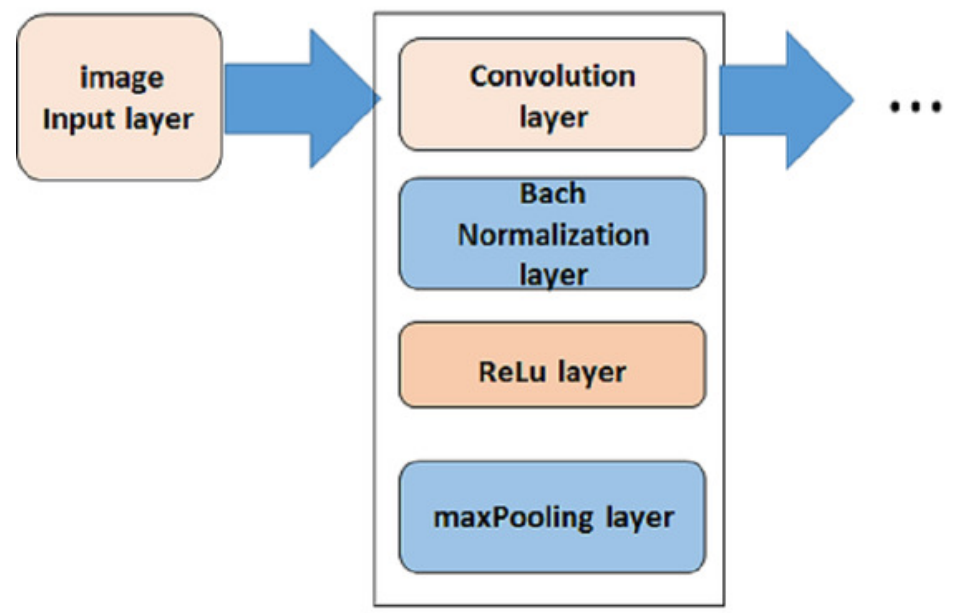

First layer

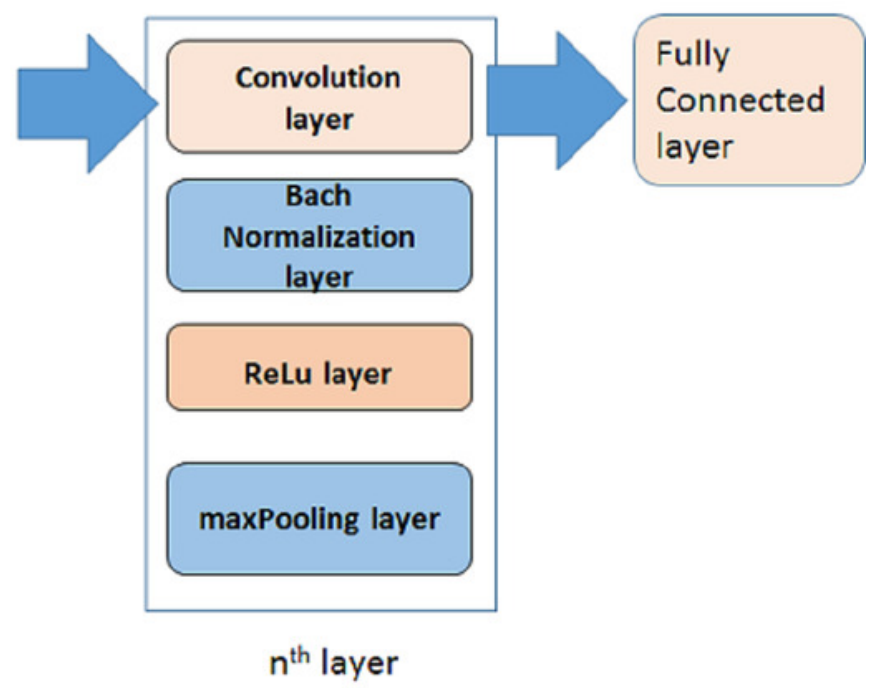


Figure 4

Training progress of running the model

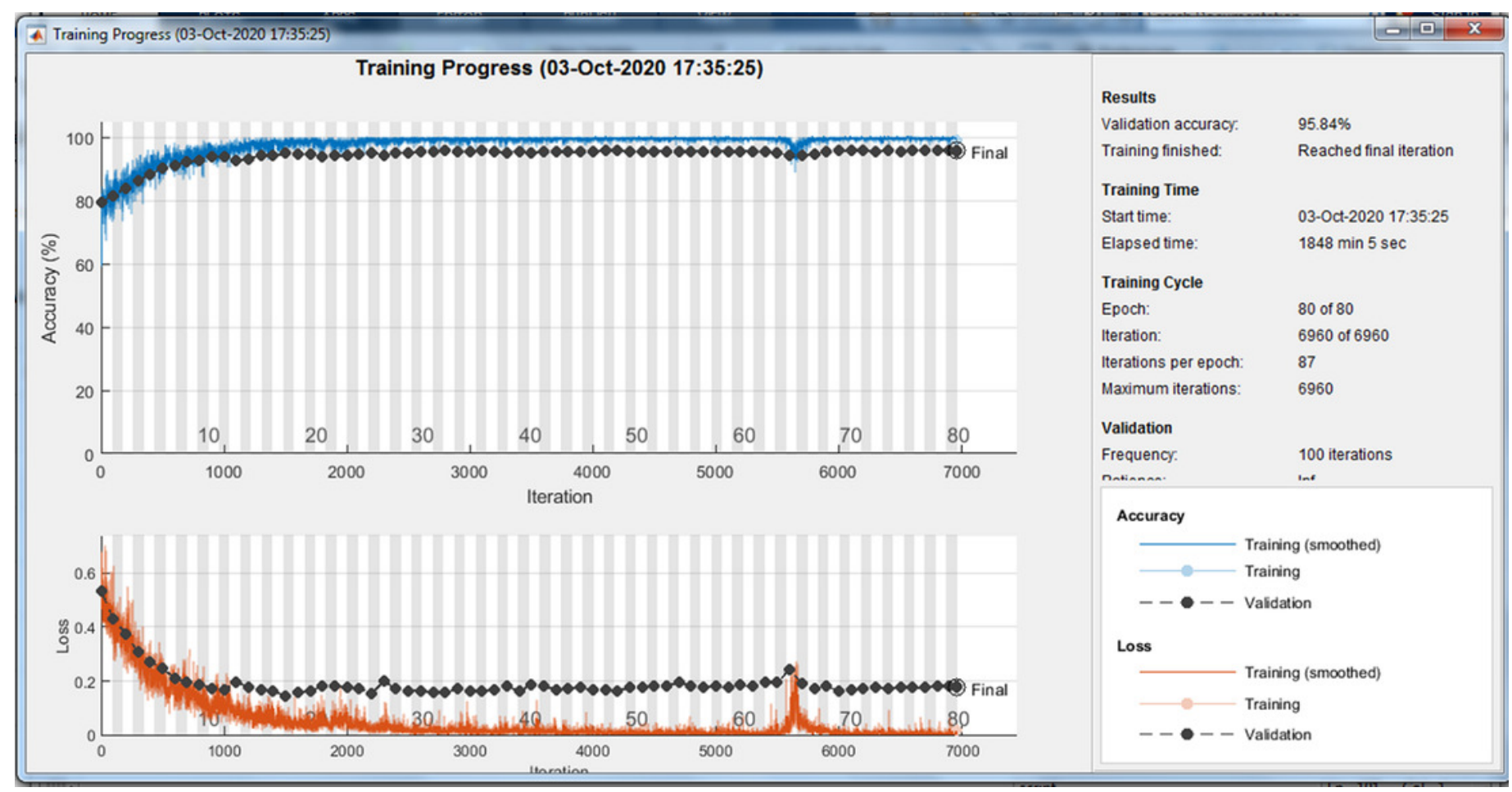




\section{Figure 5}
A) Confusion matrix definitions (Wiki., 2020), B) Confusion matrix and, C) ROC curve of evaluating training data 


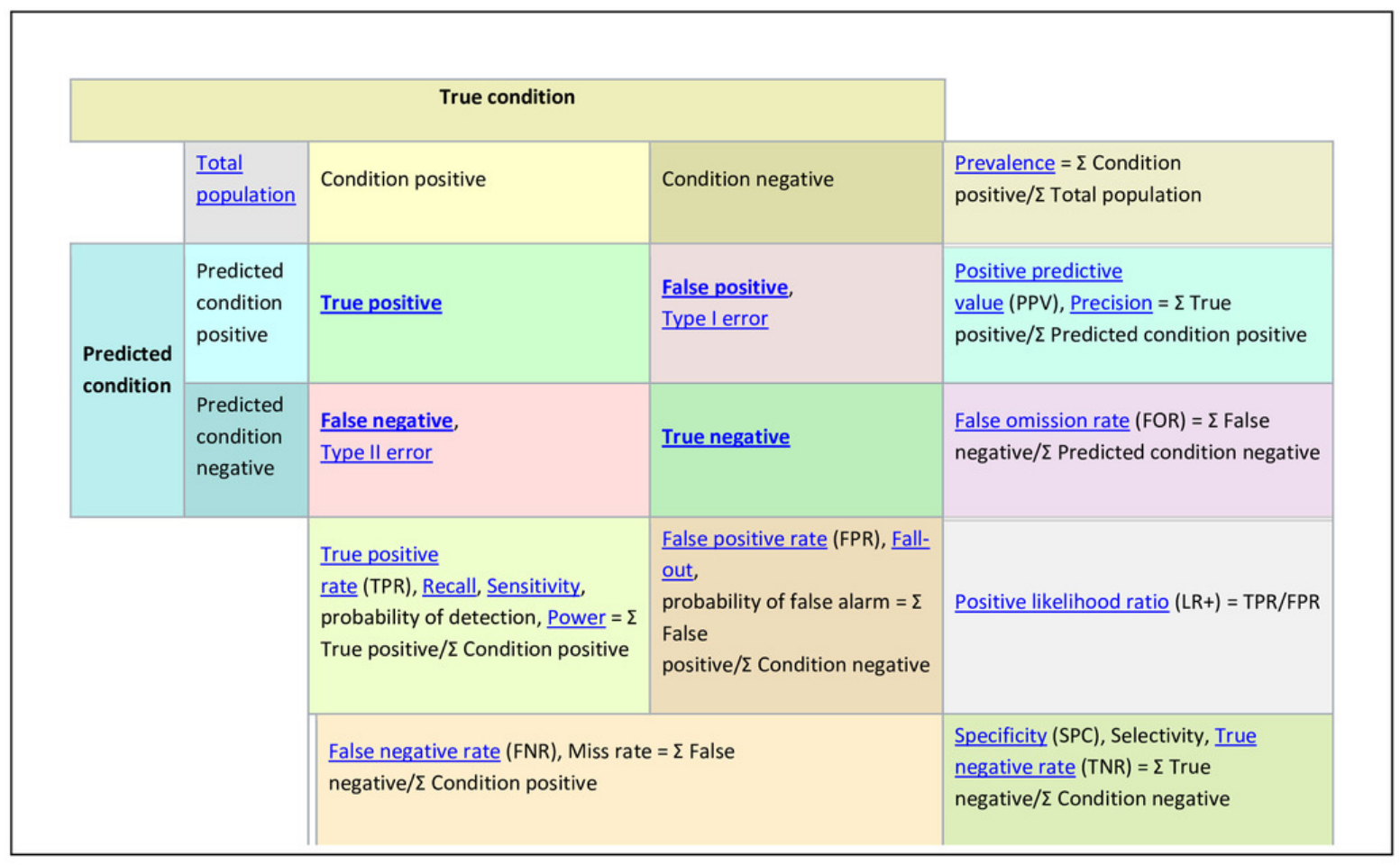

A)

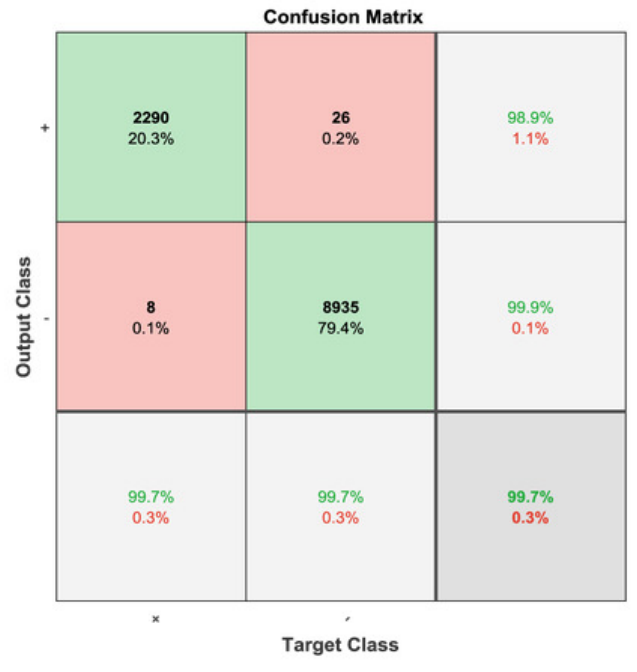

B)

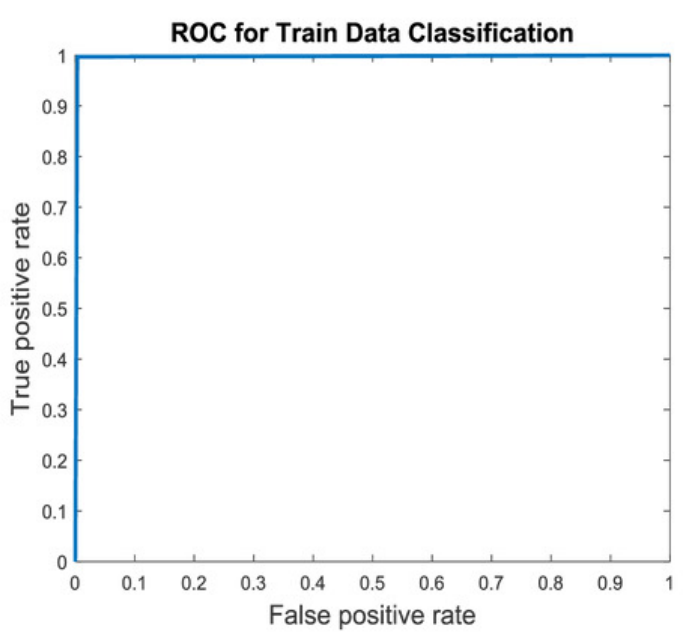

C) 
Figure 6

A) Confusion matrix and, B) ROC curve of evaluating test data 


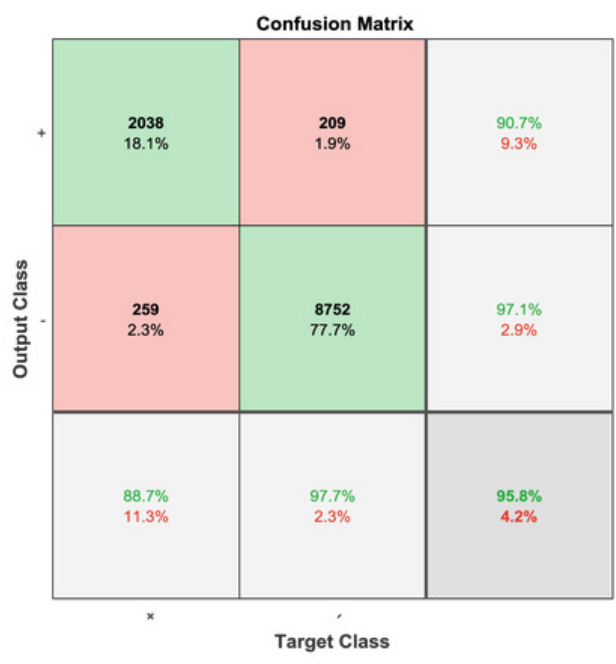

A)

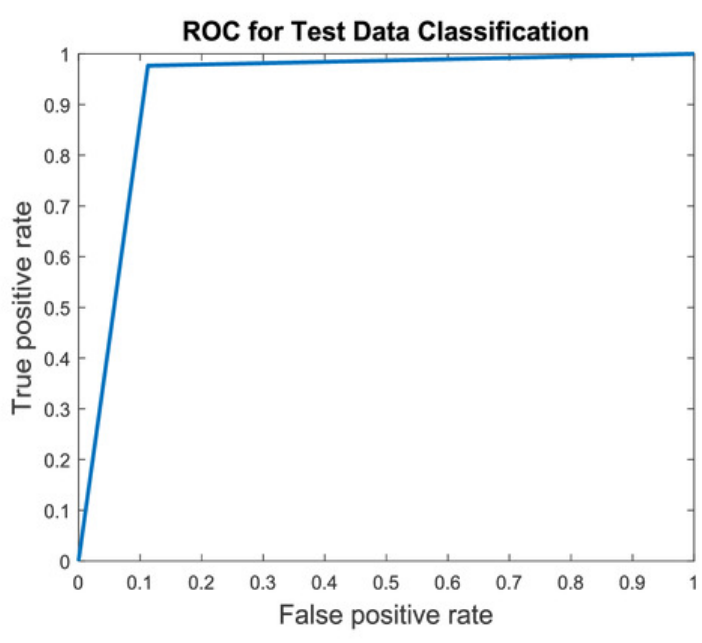

B) 


\section{Table $\mathbf{1}$ (on next page)}

Summarized results of some runs of the proposed algorithm 
Model Adjustments

\begin{tabular}{|c|c|c|c|c|c|c|c|c|c|c|}
\hline $\begin{array}{l}\text { Experi } \\
\text { ment } \\
\#\end{array}$ & Image size & $\begin{array}{l}\text { Training } \\
\text { percentage }\end{array}$ & $\begin{array}{l}\text { Max } \\
\text { Epochs }\end{array}$ & $\begin{array}{l}\text { No of } \\
\text { Conv } \\
\text { layers }\end{array}$ & $\begin{array}{l}\text { Training } \\
\text { Accuracy }\end{array}$ & $\begin{array}{l}\text { Training } \\
\text { ROC_AUC }\end{array}$ & $\begin{array}{l}\text { Test } \\
\text { Accuracy }\end{array}$ & $\begin{array}{l}\text { Test } \\
\text { ROC_AUC }\end{array}$ & $\begin{array}{l}\text { All Data } \\
\text { Accuracy }\end{array}$ & $\begin{array}{l}\text { All Data } \\
\text { ROC_AUC }\end{array}$ \\
\hline 1 & $200 * 200$ & $\begin{array}{c}50 \% \\
\text { random }\end{array}$ & 80 & 7 & 0.997 & 0.997 & 0.958 & 0.932 & 0.978 & 0.964 \\
\hline 2 & $512 * 512$ & $\begin{array}{c}50 \% \\
\text { random }\end{array}$ & 40 & 7 & 0.995 & 0.993 & 0.948 & 0.916 & 0.972 & 0.954 \\
\hline 3 & $512 * 512$ & $\begin{array}{l}50 \% \\
\text { random }\end{array}$ & 80 & 7 & 0.997 & 0.993 & 0.952 & 0.907 & 0.975 & 0.950 \\
\hline 4 & $75^{*} 75$ & $\begin{array}{c}60 \% \\
\text { random }\end{array}$ & 40 & 6 & 0.987 & 0.979 & 0.943 & 0.908 & 0.969 & 0.951 \\
\hline 5 & $50 * 50$ & $\begin{array}{c}50 \% \\
\text { random }\end{array}$ & 40 & 5 & 0.965 & 0.937 & 0.919 & 0.858 & 0.942 & 0.898 \\
\hline $6^{*}$ & $50 * 50$ & $\begin{array}{l}50 \% \\
\text { mirror }\end{array}$ & 40 & 5 & 0.975 & 0.947 & 0.916 & 0.850 & 0.945 & 0.898 \\
\hline
\end{tabular}

1 